\title{
What Do Rain Gauges Tell Us about the Limits of Precipitation Predictability?*
}

\author{
Dan Gianotti, Bruce T. Anderson, And Guido D. SAlvucci \\ Department of Earth and Environment, Boston University, Boston, Massachusetts
}

(Manuscript received 4 October 2012, in final form 26 April 2013)

\begin{abstract}
A generalizable method is presented for establishing the potential predictability for seasonal precipitation occurrence using rain gauge data. This method provides an observationally based upper limit for potential predictability for 774 weather stations in the contiguous United States. It is found that the potentially predictable fraction varies seasonally and spatially, and that on average $30 \%$ of year-to-year seasonal variability is potentially explained by predictable climate processes. Potential predictability is generally highest in winter, appears to be enhanced by orography and land surface coupling, and is lowest (stochastic variance is highest) along the Pacific coast. These results depict "hot" spots of climate variability, for use in guiding regional climate forecasting and in uncovering processes driving climate. Identified "cold" spots are equally useful in guiding future studies as predictable climate signals in these areas will likely be undetectable.
\end{abstract}

\section{Introduction}

It has long been known that Earth's atmosphere is not entirely predictable in practical or theoretical terms (Lorenz 1973; Leith 1978), but many climate processes do display a predictability that we can exploit in creating forecasts, discovering trends, and uncovering mechanisms of climate variability (Lorenz 1973; Leith 1978; Kirtman and Schopf 1998). These forecasts of climate hinge on determining future probability distributions conditioned on the present and past (Palmer 2006; NRC 2010; DelSole and Tippett 2007) and are based on uncovering variability caused by low-frequency processes that have persistence beyond the chaotic time scale of weather (Leith 1978; Palmer 2006; NRC 2010). Many of these low-frequency processes [El Niño-Southern Oscillation (ENSO), Madden-Julian oscillation, and longterm trends] are regularly used in forecasting climate variables on seasonal-to-decadal time scales (Palmer 2006; NRC 2010), while other potential predictors may remain undiscovered and unutilized.

\footnotetext{
* Supplemental information related to this paper is available at the Journals Online website: http://dx.doi.org/10.1175/JCLI-D-1200718.s1.

Corresponding author address: Dan Gianotti, Boston University, Department of Earth and Environment, 685 Commonwealth Ave., Boston, MA 02215.

E-mail: gianotti@bu.edu
}

The chaotic nature of atmospheric dynamics limits the predictability of climate variables, although the limits have not been quantified (DelSole and Tippett 2007) and have even been proposed to be undeterminable (NRC 2010). In this paper we estimate these limitstermed the potential predictability (PP) - for a single climate variable, seasonal precipitation occurrence, with the method generalizable to other variables.

Precipitation occurrence is of interest for a number of reasons. In particular, 1) variability in the number of rain events is a driver of extreme hydrological events (droughts and flooding) (Sun et al. 2006), 2) the major climate trends in U.S. precipitation have been shown to be due to an increase (or decrease) in the number of rain events as much as by major changes in precipitation intensity (Karl and Knight 1998), and 3) our tools for decadal- and century-scale forecasting (coupled global climate models) have had difficulty representing the actual variability of precipitation occurrence (the "constant drizzle" problem; Sun et al. 2006; Dai 2006).

Ferguson et al. (2011) have shown that-at least for general circulation model ensemble data-the slowly varying, boundary-induced precipitation signal is generally nonstationary over the twentieth century, while the stochastic component of precipitation variability is stationary. This suggests the idea of separating variance caused by short-memory (high frequency) processes and longer (low frequency) oscillations associated with potentially predictable processes. Because of this, if we model precipitation as an interannually stationary process, we 
would expect to represent the high-frequency ("stochastic") variability well, but not represent any of the low-frequency (potentially predictable) variability; differences between the (modeled) stochastic variability and the (observed) total variability should give an estimate of the potentially predictable variability (Katz and Parlange 1998; Madden and Shea 1978; Wang et al. 2006).

\section{Methods}

One means of determining an upper bound for the predictability of a particular variable is to establish what fraction of the variability is due to long- versus shortterm processes (Katz and Parlange 1998). Here, we establish this fraction using variable-complexity stochastic models trained on the historic record to simulate precipitation occurrence (Katz 1977). Although not all lowfrequency variability in the historic record is predictable, we can estimate what portion of the signal is attributable to (nonpredictable) short-term dynamics through comparison with an entirely high-frequency data-generating process. Our models only incorporate short-memory information (and match the observed short time-scale statistics identically), so that no low-frequency (climate) variability is introduced. To determine the appropriate complexity of the models, we use the corrected Akaike information criterion $\mathrm{AIC}_{c}$ to optimize the tradeoff between goodness-of-fit and parsimony/complexity (Hurvich and Tsai 1989). We then use the models to simulate 1000 realizations of each station's roughly 100 -yr historic record, which form a distribution of purely stochastic simulated precipitation records for a given station.

To compare the observed record with the simulated records, we use the number of days of precipitation within a centered seasonal (89 day) window. Although any window could be selected, the seasonal window establishes a predictability metric of appropriate scale for use in assessing local droughts (Ferguson et al. 2011), agricultural growing periods (D'Odorico et al. 2000), or winter snowpack (Bohr and Aguado 2001) and still allows us some insight into the seasonal cycle of PP throughout the year.

Potential predictability of observed precipitation frequency at a given station is defined as the portion of variability that cannot be explained by the stochastic evolution of daily precipitation events-an unexplained variance-usually as a normalized ratio. Although sometimes expressed as a signal-to-noise ratio (Ferguson et al. 2011; Leith 1978) or a signal-toforecast-error ratio (Somerville 1987), we use the unexplained variance fraction definition (Boer 2004; Rowell 1998):

$$
\mathbf{P P}_{89}=\frac{\boldsymbol{\sigma}_{89, \mathrm{obs}}^{2}-\left\langle\boldsymbol{\sigma}_{89, \mathrm{sim}}^{2}\right\rangle}{\boldsymbol{\sigma}_{89, \mathrm{obs}}^{2}},
$$

where $\mathbf{P P}_{89}$ is the potential predictability for a seasonal 89-day window centered on calendar day $t, \boldsymbol{\sigma}_{89, \mathrm{obs}}^{2}$ is the variance (between years) in the number of rainy days within an 89-day window of $t( \pm 44)$ in the observed record, and $\left\langle\boldsymbol{\sigma}_{89, \text { sim }}^{2}\right\rangle$ is the mean variance (between years) in the number of rainy days within an 89-day window of $t$ in the 1000 simulated records. The numerator represents the variance not explained by the stationary stochastic model and is expressed as a fraction of the total (observed) variance from the historical record. Positive values of $\mathbf{P P}_{89}$ correspond to values of $\boldsymbol{\sigma}_{89, \mathrm{obs}}^{2}$ in the positive tail of the $\boldsymbol{\sigma}_{89 \text { sim }}^{2}$ distribution; the higher the value of $\mathbf{P} \mathbf{P}_{89}$, the less likely it is that the observed variance is due the integration of random short-memory processes (i.e., is due to chance).

Our models and analysis are based on the U.S. Historical Climatology Network (USHCN) dataset (Williams et al. 2006) using daily precipitation values through 31 December 2009. Of the 1218 available weather stations in the USHCN, a subgroup of 774 stations is selected to meet longevity and completeness criteria (see the supplemental material available online at http://dx.doi.org/ 10.1175/ JCLI-D-12-00718.s1). All stations are required to have at least 80 years of continuous data (from 1 January 1930 to 31 December 2009) and cannot have more than $5 \%$ of daily values missing. The longest period ending on 31 December 2009 with at least $95 \%$ complete data is selected. The threshold for precipitation occurrence is $0.254 \mathrm{~mm}$ ( $0.01 \mathrm{in}$.); climatology of precipitation occurrence from these data is shown in Figs. 1a-d.

Markov chain models are well suited for stochastic precipitation occurrence modeling (Katz 1977), and model complexity can be selected by allowing for a variable chain order $m$. Each state of the Markov chain is represented by the day of the year and occurrence pattern over the previous $m$ days (Wilks and Wilby 1999). The transition probabilities- the probabilities of precipitation on a certain day given the last $m$ days' history-are found empirically from the historic record for each possible occurrence pattern on each day of the year.

Our method for determining seasonal PP values is shown schematically in supplemental Fig. S1. Models are created to match the empirical historic record for each of 774 selected weather stations across the United States; for each station, the order $m$ is established by using the $\mathrm{AIC}_{c}$ (see the supplemental material) and is allowed to vary from day to day (Hurvich and Tsai 1989; Katz and Parlange 1993). The $\mathrm{AIC}_{c}$ is used because it 

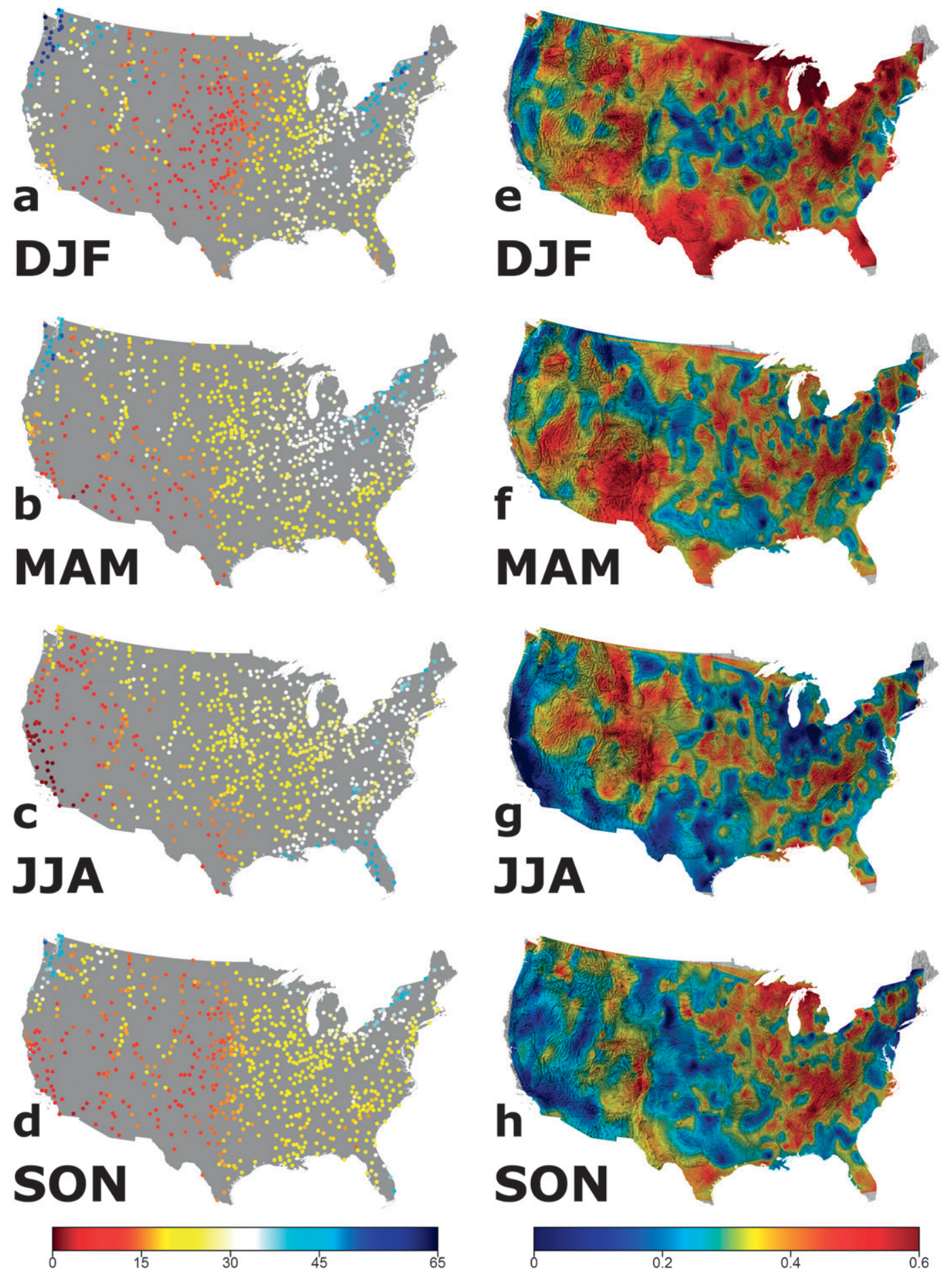

FIG. 1. Climatology and PP of precipitation occurrence. (a)-(d) The mean number of days with precipitation $>0.254 \mathrm{~mm}$ (0.01 in.) for 89-day seasonal windows centered on 15 January, 15 April, 15 July, and 15 October, respectively. Circles show the location of the 774 weather stations used in the study. (e)-(h) PP as calculated by $\left(\boldsymbol{\sigma}_{89, \mathrm{obs}}^{2}-\left\langle\boldsymbol{\sigma}_{89, \mathrm{sim}}^{2}\right\rangle\right) / \boldsymbol{\sigma}_{89, \mathrm{obs}}^{2}$. The Appalachians, Southern Rockies, and Great Lakes regions show the highest PP, while high wintertime stochastic variability in the Northwest and on the Pacific coast lead to a PP below the national average. See supplemental Fig. S3 for timing of min and max PP and supplemental Fig. S5 for spatial significance. 
allows for an unbiased optimization of the tradeoff between model complexity/parsimony (using a higher order in determining the transition probabilities is more complex and introduces more variability) and model likelihood (higher-order models increase the likelihoodbased fit). We also allow pooling of our dataset (using multiple neighboring days' observations for fitting model transition probabilities - see the supplemental material), again using the $\mathrm{AIC}_{c}$ to determine appropriate pooling windows.

Once the pooling size, daily chain orders, and transition probabilities are determined, the stochastic model for that station is complete, and simulations can be created where each day's occurrence is determined probabilistically based on the previous $m$ days' occurrence history. We simulate 1000 realizations of each station's roughly 100 -yr historic record $\left(\sim 3 \times 10^{10}\right.$ individual days of precipitation occurrence in total), which then form a distribution of purely stochastic simulated precipitation records for a given station.

To analyze the spatial patterns of predictability, we grid the $\mathbf{P P}_{89}(t)$ vector $(t \in\{1,2, \ldots, 365\})$ at $0.0775^{\circ}$ resolution using natural neighbor interpolation (Sibson 1981). To create clusters we use the $k$-means algorithm (MacQueen 1967) with an L2 norm (squared Euclidean distance) to group the clusters so that moving any one grid point to another cluster increases the mean intracluster distance.

\section{Results}

The maps shown in Figs. 1e-h indicate areas of high and low PP by season, with considerable variability observed in both magnitude and spatial pattern throughout the year. This figure is intended to serve as a "road map" for areas with high and low likelihoods for success in interannual- to multidecadal-scale modeling of precipitation occurrence, corresponding to high- and low-PP values, respectively.

In all of the maps in Figs. 1e-h there are clear correlations between high PP and orographic features, most notably in the Southern Rockies and Appalachians. This suggests that orography may play a role in amplifying not just spatial but temporal precipitation variability (and thereby PP), presumably by amplifying temporal variations in local water vapor convergence (storm tracks and convection). The Pacific coast is noteworthy in its relatively low PP, due primarily to very low observed variability in the summer and fall (Figs. 1g,h) and relatively high stochastic variability in the fall through spring (Figs. 1e,g,h; see also supplemental Fig. S2). The mean PP value across all stations and days of the year is 0.3 (30\% of year-to-year seasonal variability is potentially explained by predictable climate processes), although at some stations the seasonal-mean PP exceeds 0.7. All PP values above 0.24 are significant at the 0.05 confidence level; those below 0.24 are either significant (but low) or indistinguishable from zero (see the supplemental material).

It is important to note that low PP does not necessarily mean that predictable processes have little influence in a region. Rather, it suggests that the predictable portion of the interannual variance in number of rainy days in a season is small relative to the high-frequency-driven stochastic variability that the area experiences.

To attribute climate processes to their effects on specific climate variables, we hope to find common regional, seasonal behavior patterns. To this end, we performed a $k$-means cluster analysis based on an evenly gridded set of PP values and plotted the resulting regional patterns (Fig. 2). Although implicitly dependent on spatial patterns to some degree through the gridding process, the clustering is otherwise independent of location and based only on similarities in the seasonal pattern of PP. The overall 774 station-mean pattern shows higher predictability in the late fall and winter, and lower predictability in the summer and early fall, confirming an earlier result by Rowell (1998).

Cluster 1 has higher-than-spatial-mean predictability in summer, lower in fall and winter, which can also be thought of as a "flattening" of the seasonal predictability cycle. This group of stations has the highest relative summer PP, which agrees well with previous studies on the strong JJA soil-moisture convective precipitation feedbacks in the western Great Plains and Ozarks/Gulf Coast (Koster et al. 2003), driven, at least in part, by sea surface temperature (SST) variability and atmospheric water vapor transport from the Gulf of Mexico (Wang et al. 2010). In addition, Koster et al. (2004) suggest that this feedback-in which local soil moisture enhances local evaporation and precipitationshould happen in transition areas from wet to dry climates, further supporting a soil moisture explanation for enhanced summertime predictability in the western portion of cluster 1 .

Cluster 2 displays an amplification of the spatial average predictability cycle, with high late-fall and winter PP. The generally high PP in the Great Lakes and Finger Lakes regions suggests a PP amplification during the lake-effect snow period from October through midMarch (Niziol et al. 1995), with the available water supply likely amplifying the predictability of temporal patterns in atmospheric water vapor. Although the mechanisms are likely different, Texas and Florida have similar seasonal patterns to the Great Lakes region because of their strong correlation with tropical Pacific 


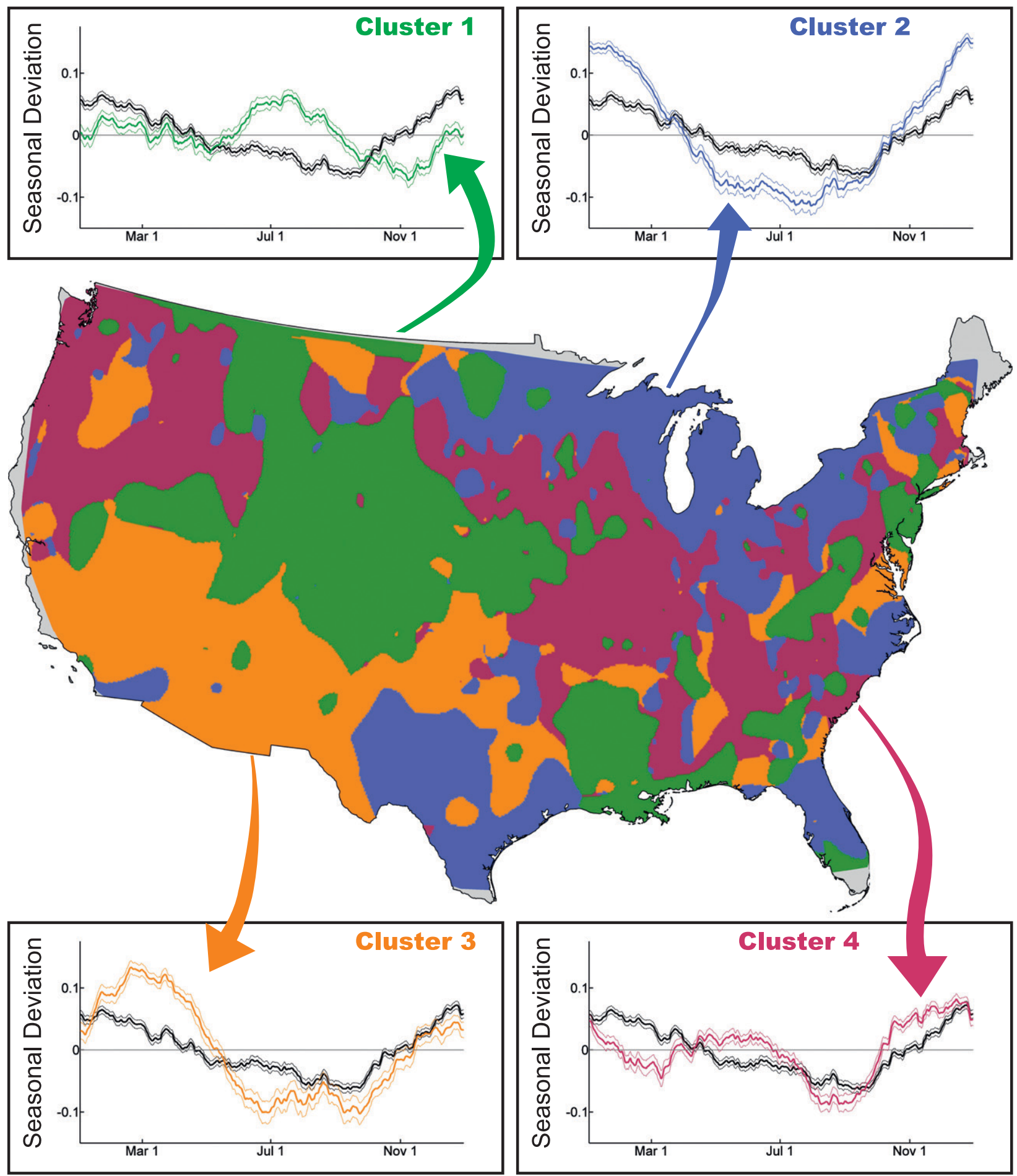

FIG. 2. Cluster analysis based on potential predictability seasonal time series. Regions determined by an interpolated L2-norm clustering algorithm performed on the seasonal predictability curves. Subplots show the mean seasonal anomaly curve \pm 1.96 standard error (SE) over all 774 stations (black) and the mean anomaly curve \pm 1.96 SE for the cluster (color). Clusters were determined using $k$-means on a gridded PP field, and cluster curves are the mean of the station values in that region (rather than the mean of the gridded values). 
SSTs from December through March (Boer 2004; Ropelewski and Halpert 1986).

Cluster 3-located mostly in the Southwest-has a seasonal predictability cycle that generally follows the spatial average with the exception of late winter, when predictability is significantly higher. Although coastal southern California has, on average throughout the year, a much lower predictability than the Colorado Plateau to the east (Fig. 2), the region as a whole lies along the ENSO precipitation anomaly track (Cayan et al. 1999), and as such the predictability peaks for stations in this region are representative of the typical late-winter ENSO precipitation timings (McCabe and Dettinger 1999).

Cluster 4 is best described as a flattening of the mean PP cycle from January through June with a PP increase in October and November. Although wintertime precipitation in the Pacific Northwest has been shown to be strongly affected by ENSO variability, we find that the predictable variability in precipitation occurrence is minimal relative to winter in the Southwest, in agreement with previous results (Feldl and Roe 2011). As previously mentioned, this does not mean that ENSO does not affect Northwest precipitation occurrence, but that the predictable fraction of the variance is small relative to the stochastic component (which is in fact higher in the Northwest than any other region; see supplemental Fig. S2).

\section{Discussion}

All PP is due to the variability of processes leading to precipitation, and the timing/magnitude of the PP response is a function of interactions between PP drivers [SSTs (Cayan et al. 1998) and anthropogenic forcing (Zhang et al. 2007)], feedbacks [soil-moisture (Koster et al. 2003, 2004) and orography], and unpredictable dynamics. Rigorous attribution of PP-related precipitation processes to oscillations, trends, and feedbacks is likely best targeted toward areas of high PP-the Appalachians, Rockies, and Great Lakes, for instanceusing Fig. 1 as a guide for region selection and Fig. 2 as a guide for timings and likely processes. Further, the method for determining PP discussed here is algorithmic and generalizable to climate variables other than precipitation occurrence, which suggests a high-level approach to climate forecasting - namely, to 1) establish the magnitudes of predictable and stochastic variances to determine PP, 2) look for processes that drive variability in high-PP regions to close the gap between current model skill and PP limits, 3) use the findings from regional process modeling to update global models and forecasts, and 4) monitor areas for changes in PP over time that might signify changes in predictable climate processes.
Acknowledgments. This work was supported by the National Science Foundation (AGS-0958907) and U.S. Department of Energy (DE-SC0006914). We extend our thanks to Toby Fusco for compiling the station data and to Indrani Pal, Randy Koster, and one anonymous reviewer for their helpful feedback.

\section{REFERENCES}

Boer, G., 2004: Long time-scale potential predictability in an ensemble of coupled climate models. Climate Dyn., 23, 29-44.

Bohr, G., and E. Aguado, 2001: Use of April 1 SWE measurements as estimates of peak seasonal snowpack and total cold-season precipitation. Water Resour. Res., 37, 51-60.

Cayan, D., M. Dettinger, H. Diaz, and N. Graham, 1998: Decadal variability of precipitation over western North America. J. Climate, 11, 3148-3166.

—, K. Redmond, and L. Riddle, 1999: ENSO and hydrologic extremes in the western United States. J. Climate, 12, 28812893.

Dai, A., 2006: Precipitation characteristics in eighteen coupled climate models. J. Climate, 19, 4605-4630.

DelSole, T., and M. Tippett, 2007: Predictability: Recent insights from information theory. Rev. Geophys., 45, RG4002, doi:10.1029/2006RG000202.

D'Odorico, P., L. Ridolfi, A. Porporato, and I. Rodriguez-Iturbe, 2000: Preferential states of seasonal soil moisture: The impact of climate fluctuations. Water Resour. Res., 36, 2209-2219.

Feldl, N., and G. H. Roe, 2011: Climate variability and the shape of daily precipitation: A case study of ENSO and the American West. J. Climate, 24, 2483-2499.

Ferguson, I., P. Duffy, T. Phillips, X. Liang, J. Dracup, S. Schubert, and P. Pegion, 2011: Non-stationarity of the signal and noise characteristics of seasonal precipitation anomalies. Climate Dyn., 36, 739-752.

Hurvich, C. M., and C.-L. Tsai, 1989: Regression and time series model selection in small samples. Biometrika, 76, 297-307.

Karl, T., and R. Knight, 1998: Secular trends of precipitation amount, frequency, and intensity in the United States. Bull. Amer. Meteor. Soc., 79, 231-241.

Katz, R., 1977: Precipitation as a chain-dependent process. J. Appl. Meteor., 16, 671-676.

, and M. Parlange, 1993: Effects of an index of atmospheric circulation on stochastic properties of precipitation. Water Resour. Res., 29, 2335-2344.

_ modeling of precipitation. J. Climate, 11, 591-601.

Kirtman, B., and P. Schopf, 1998: Decadal variability in ENSO predictability and prediction. J. Climate, 11, 2804-2822.

Koster, R., M. Suarez, R. Higgins, and H. Van den Dool, 2003: Observational evidence that soil moisture variations affect precipitation. Geophys. Res. Lett., 30, 1241, doi:10.1029/2002GL016571.

, and Coauthors, 2004: Regions of strong coupling between soil moisture and precipitation. Science, 305, 1138-1140.

Leith, C., 1978: Predictability of climate. Nature, 276, 352-355.

Lorenz, E. N., 1973: Predictability and periodicity: A review and extension. Proc. Third Conf. on Predictability and Statistics in the Atmospheric Sciences, Boulder, CO, Amer. Meteor. Soc., 1-4.

MacQueen, J., 1967: Some methods for classification and analysis of multivariate observations. Proc. Fifth Berkeley Symp. on Mathematical Statistics and Probability, Vol. 1, Berkeley, CA, University of California, 281-297. 
Madden, R., and D. Shea, 1978: Estimates of the natural variability of time-averaged temperatures over the United States. Mon. Wea. Rev., 106, 1695-1703.

McCabe, G., and M. Dettinger, 1999: Decadal variations in the strength of ENSO teleconnections with precipitation in the western United States. Int. J. Climatol., 19, 1399-1410.

Niziol, T., W. Snyder, and J. Waldstreicher, 1995: Winter weather forecasting throughout the eastern United States. Part IV: Lake effect snow. Wea. Forecasting, 10, 61-77.

NRC, 2010: Assessment of intraseasonal to interannual climate prediction and predictability. National Research Council, 173 pp.

Palmer, T., 2006: Predictability of weather and climate: From theory to practice. Predictability of Weather and Climate, Cambridge University Press, 1-29.

Ropelewski, C. F., and M. S. Halpert, 1986: North American precipitation and temperature patterns associated with the El Niño-Southern Oscillation (ENSO). Mon. Wea. Rev., 114, 2352-2362.

Rowell, D., 1998: Assessing potential seasonal predictability with an ensemble of multidecadal GCM simulations. J. Climate, 11, 109-120.

Sibson, R., 1981: A brief description of natural neighbor interpolation. Interpreting Multivariate Data, V. Barnett, Ed., Wiley, 21-36.
Somerville, R., 1987: The predictability of weather and climate. Climatic Change, 11, 239-246.

Sun, Y., S. Solomon, A. Dai, and R. Portmann, 2006: How often does it rain? J. Climate, 19, 916-934.

Wang, H., S. Schubert, M. Suarez, and R. Koster, 2010: The physical mechanisms by which the leading patterns of SST variability impact U.S. precipitation. J. Climate, 23, 1815-1836.

Wang, J., B. Anderson, and G. Salvucci, 2006: Stochastic modeling of daily summertime rainfall over the southwestern United States. Part I: Interannual variability. J. Hydrometeor., 7, 739754.

Wilks, D., and R. Wilby, 1999: The weather generation game: A review of stochastic weather models. Prog. Phys. Geogr., 23, 329-357.

Williams, C. N., R. S. Vose, D. R. Easterling, and M. J. Menne, 2006: United States historical climatology network daily temperature, precipitation, and snow data. Carbon Dioxide Information Analysis Center, Oak Ridge National Laboratory, Oak Ridge, Tennessee. [Available online at http://cdiac. ornl.gov/ftp/ushcn_daily/.]

Zhang, X., F. Zwiers, G. Hegerl, F. Lambert, N. Gillett, S. Solomon, P. Stott, and T. Nozawa, 2007: Detection of human influence on twentieth-century precipitation trends. Nature, 448, $461-465$. 